\title{
Taxonomia corporativa e taxonomia facetada: usos e aplicações na ciência da informação no Brasil
}

\author{
Pâmela Tamires Dias Lopes* \\ Elisângela Cristina Aganette* \\ Benildes Coura M. S. Maculan*
}

Artículo recibido:

3 de diciembre de 2018

Artículo aceptado:

24 de mayo de 2019

Artículo de investigación

\section{Resumo}

As taxonomias permitem uma recuperação da informação mais precisa, pois direciona o usuário na busca, por meio dos rótulos das classes e subclasses presentes na estrutura hierárquica. $\mathrm{O}$ objetivo do presente artigo é apresentar resultado parcial de uma pesquisa mais ampla, oferecendo os usos e aplicações de taxonomias corporativas e facetadas como instrumentos de representação da informação e do conhecimento, descritas em teses e dissertações no âmbito da Ciência da Informação. A metodologia incluiu um levantamento bibliográfico na Biblioteca Digital de Teses e Dissertações da Capes, para mapear as teses e dissertações relacionadas

* Escola de Ciência da Informação (ECI) da Universidade Federal de Minas Gerais (UFMG), Brasil

pamela.td143@gmail.com elisangelaaganette@gmail.com benildes@gmail.com

INVESTIGACIÓN BIBLIOTECOLÓGICA, vol. 34, núm. 82, enero/marzo, 2020, México, ISSN: 2448-8321 pp. 159-173 
à temática. Foram recuperadas apenas sete dissertações na Ciência da Informação, no período de 15 anos, e nenhuma tese. A análise dos documentos utilizou o método da Análise de Conteúdo, a partir das categorias: objetivo, metodologia, produto final e trabalhos futuros. Os resultados mostraram o estado da arte dos usos e aplicações dos instrumentos investigados, assim como as tendências para estudos futuros. O quantitativo pouco representativo de pesquisas evidencia a necessidade de mais estudos envolvendo esses instrumentos.

Palavras-chave: Taxonomia Corporativa; Taxonomia Facetada; Usos e Aplicações; Ciência da Informação

Taxonomía corporativa y taxonomía facetada: sus usos y aplicaciones en las ciencias de la información del Brasil

Pâmela Tamires Dias Lopes, Elisângela Cristina Aganette y Benildes Coura M. S. Maculan

\section{RESUMEN}

Las taxonomías permiten una recuperación más precisa de la información, ya que ayudan al usuario a buscar a través de las etiquetas de las clases y subclases presentes en la estructura jerárquica. El objetivo de este artículo es presentar un resultado parcial de una investigación más amplia, ofreciendo los usos y aplicaciones de las taxonomías corporativas y facetadas como instrumentos de representación de la información y el conocimiento, descritos en trabajos académicos en el campo de las ciencias de la información. La metodología incluyó una encuesta bibliográfica en la Biblioteca Digital de Teses e Dissertações de Capes, para mapear las tesis y tesis de posgrado relacionadas con el tema. Sólo se recuperaron siete tesis de posgrado en ciencias de la información en el periodo de 15 años, y ninguna tesis. En el análisis de los documentos se utilizó el método de análisis de contenidos, a partir de las categorías objetivo, metodología, producto final y trabajos futuros. Los resultados mostraron el estado del arte de los usos y aplicaciones de los instrumentos investigados, así como las tendencias para estudios futuros. La pequeña cantidad de investigaciones evidenció la necesidad de realizar más estudios que involucren estos instrumentos. 
Palabras clave: Taxonomía Corporativa; Taxonomía Facetada; Usos y Aplicaciones; Ciencias de la Información

Corporate and faceted taxonomies: analysis of its uses and applications in the information science of Brazil

Pâmela Tamires Dias Lopes, Elisângela Cristina Aganette and Benildes Coura M.S. Maculan

\section{Abstract}

Taxonomies allow a more accurate retrieval of information, since they direct the user to the search, through the labels of the classes and subclasses present in the hierarchical structure. The purpose of this article is to present a partial result of a broader research, offering the uses and applications of corporate and face taxonomies as instruments of information and knowledge representation, described in theses and dissertations in the field of Information Science. The methodology included a bibliographic survey in the Biblioteca Digital de Teses e Dissertações of Capes, in order to map the theses and dissertations related to the subject. Only seven dissertations in Information Science were retrieved over a period of 15 years, and not even one thesis. The exploration of the documents used the Content Analysis method, based on the categories: objective, methodology, final product and future work. The results showed the state of the art over the uses and applications of the investigated instruments, as well as the trends for future studies. The non-representative quantitative of the research evidenced the need for more studies involving these instruments.

Keywords: Corporate Taxonomy; Faceted Taxonomy; Uses and Applications; Information Science

\section{INTRODUÇÃO}

\footnotetext{
Arganização e representação da informação e do conhecimento posIsuem grande relevância para tomadas de decisão, por isso faz-se necessário à diversidade de sistemas que de fato organizem e representem o
} 
conhecimento e a informação existente e utilizadas para sanar diferentes necessidades de recuperação e uso da informação. Com o intuito de possibilitar uma melhor recuperação e acesso à informação os profissionais fazem uso de diversas ferramentas de representação e organização, para assim, facilitar o acesso às mesmas. Os instrumentos de representação e organização do conhecimento e da informação surgem como uma forma de compartilhar as informações a partir de um único ambiente e, mais do que isso, são considerados ferramentas de apoio à gestão do conhecimento, cuja finalidade não consiste apenas em disponibilizar informações, mas principalmente em compartilhar e gerar novos conhecimentos (Aganette, 2010). Dentre as ferramentas utilizadas estão às taxonomias, objeto de estudo do presente artigo. As taxonomias são estudadas em diferentes campos do conhecimento e com distintos propósitos. Neste artigo interessam as taxonomias corporativas e facetadas empregadas como instrumento de organização do conhecimento e da informação, estudadas, utilizadas e aplicadas na CI (Ciência da Informação).

Conceitualmente, as taxonomias possuem várias e diferentes definições, características, origens e usos práticos em diferentes ambientes informacionais, porém, algumas são reincidentes, e evidenciadas por diferentes autores. Uma delas refere-se à construção sistemática e à ordenação da informação a partir de sua estrutura hierárquica de subordinação de assuntos (Aganette, 2010). Pode-se afirmar que uma taxonomia é um modo de organizar, classificar e agrupar coisas, por meio de atributos específicos, em seguida, atribuir nomes a esses grupos de coisas.

Diante do crescente volume de informação, principalmente no contexto web, os meios para a representação e organização do conhecimento e da informação precisam de aprimoramento, com a finalidade de suprir a necessidade de se potencializar os instrumentos que auxiliem na representação e organização informacional. Faz-se necessário, maiores pesquisas e estudos referentes aos meios de representação e organização do conhecimento e da informação.

Por meio deste estudo, evidenciou-se que as taxonomias corporativas e as taxonomias facetadas são tratadas com maior ênfase na área da CI. O estudo evidenciou, ainda, uma lacuna na literatura da área sobre o estado da arte em relação aos conceitos, usos e aplicações desses dois tipos de taxonomias. Assim, a partir dessa lacuna, decidiu-se por explorar e mapear os usos e aplicações desses dois tipos de taxonomias nas produções acadêmicas refletidas nas teses e dissertações defendidas na CI. Procura-se averiguar para qual direção os trabalhos nas áreas estão apontando, em especial para os usos e aplicações das taxonomias.

O presente trabalho tem como objetivo, mapear os usos e as aplicações de taxonomias corporativas e facetadas na Ciência da Informação, com o 
intuito de investigar as abordagens apresentadas nos trabalhos, explorando o estado da arte das temáticas envolvidas nas investigações stricto sensu. Para tanto, este artigo, após essa introdução, se organiza da seguinte maneira: a segunda seção apresenta o background da pesquisa, com a caracterização dos documentos do tipo dissertações e teses, que foram analisados, assim como a apresentação dos conceitos de taxonomia corporativa e taxonomia facetada como instrumento de representação da informação e do conhecimento na Ciência da Informação; a terceira seção descreve a metodologia empregada, seguida da quarta seção, que apresenta os resultados; finalmente, as considerações finais.

\section{BACKGROUND}

\section{Teses e dissertaçôes}

Para Alvarenga (2000: 123), os estudos a respeito da produção e comunicação científica, podem ser ainda considerados incipientes, mesmo considerando que a produção específica neste assunto tem crescido consideravelmente. Sobre as pesquisas que visam o conhecimento dessa produção, Alvarenga (2000: 123) afirma que: "Os resultados de pesquisas, que têm por objeto a comunicação científica, podem ser vistos como subsídios para os estudos epistemológicos e arqueológicos no conhecimento de campos disciplinares constantes do sistema dos saberes. Por isso se faz necessária os cursos de Pós-Graduação e o incentivo às pesquisas”.

A partir do conteúdo que é registrado nesses documentos acadêmicos, dissertações e teses, os cursos de pós-graduação stricto sensu são uma forma de refletir sobre os esforços para se obter uma maior formação de pesquisadores, sobre o incentivo a produção e comunicação científica. Leva-se também em consideração a qualidade do curso, segundo Cendón, Kremer e Santos Campello (2000: 127)

As teses e dissertações devem ser analisadas no contexto da educação pós-graduada. Seu valor e qualidade vão depender também da qualidade dos cursos onde são produzidas, além da competência do orientador e, portanto, o processo de seleção de teses para inclusão no acervo de bibliotecas deve levar em conta esses aspectos. 
O surgimento do mestrado em CI no Brasil ocorreu no ano de 1970 e se deu em um momento peculiar, quando se estudava o Sistema Nacional de Informação em Ciência e Tecnologia (SNICT), que acabou não sendo implantado e que teve como efeito outras ações realizadas pela Organização das Nações Unidas para a Educação, a Ciência e Cultura (UNESCO). A informação vinha sendo agregada, aos poucos, nos planos governamentais brasileiros. $\mathrm{O}$ mestrado em CI, não ocorreu muito diferente aos outros cursos de pós-graduação no Brasil, que obtiveram a participação de professores estrangeiros. Em relação ao que se entende por dissertação, Cunha e Cavalcanti (2008: 130) descrevem que "Dissertação 1. Documento escrito, científico, técnico ou literário, apresentado a uma banca examinadora para o obtenção, em geral, do grau de mestre".

Finalmente em 1992 é criado o doutorado em CI, 22 anos após o início do mestrado. Esse longo período provavelmente contribuiu para que as dissertações apresentassem características de tese, quanto às exigências acadêmicas, e fez com que o tempo de sua elaboração também se prolongasse.

\section{Taxonomia corporativa e taxonomia facetada na ciência da informação}

Nos últimos tempos, as pesquisas relacionadas à recuperação da informação vêm ganhando maior destaque devido à quantidade crescente de informações disponibilizadas e pelo uso de Tecnologias de Informação e Comunicação - TIC, que estabelece interseção entre diversas áreas, tendo como exemplo a Ciência da Informação. O termo taxonomia é recente e se deu em vista da necessidade de se recuperar a informação de forma fácil e eficiente, e seu desenvolvimento e uso têm se tornado cada vez maior. As taxonomias, no ambiente corporativo são utilizadas para auxiliar na gestão da informação e do conhecimento, portanto elas são delineadas como "elementos estruturantes, estratégicos e centrais para negócios baseados em informação e conhecimento" (Terra, 2005: 1).

A taxonomia corporativa está presente em diferentes ambientes e serviços que estrutura e organiza o conhecimento registrado em poder das organizações, organiza produtos e serviços, e se constitui como um mecanismo de apoio à gestão. Ela é construída de modo a atender a um público particular, servindo de interface sistema-usuário por meio da navegação.

A taxonomia facetada emprega os princípios da Teoria da Classificação Facetada, desenvolvida por Ranganathan (1967), que foi criada com o objetivo de auxiliar na organização de materiais bibliográficos. Na construção de 
instrumentos de representação da informação e do conhecimento, a teoria fornece cinco categorias mais abstratas de mapeamento do domínio representado, formando-se múltiplas hierarquias de classes (facetas) e subclasses (subfacetas) que exprimem as diferentes dimensões desse dado domínio. Esse modo de classificação permite que o usuário encontre primeiro o assunto mais amplo, e, através dele, chegue à informação mais restrita. Essa navegação torna mais acessível e prática a recuperação da informação por parte do usuário.

\section{Procedimentos metodológicos}

Trata-se de um estudo bibliográfico de cunho qualitativo, com a finalidade de analisar os resultados de pesquisas na área da Ciência da Informação por meio dos bancos de dados de teses e dissertações. Os dados aqui analisados são parciais e fazem parte de uma pesquisa em andamento. Para a coleta dos dados, selecionou-se o Banco de Teses \& Dissertações da Capes, essa escolha se deu pelo motivo do mesma abarcar teses e dissertações de todo o país e estar em constante atualização. Os dados foram colhidos entre o dia 15 de novembro até a data de 29 de novembro de 2018.

\section{Estratégia de busca}

Para uma melhor definição de quais teses e dissertações seriam analisadas, algumas estratégias foram adotadas. Realizou-se a busca com somente termos e resultados na língua portuguesa. Para um melhor resultado, utilizou-se da busca avançada, selecionando somente a área da Ciência da Informação, com delimitação de pesquisas realizadas nos últimos 15 anos. Durante o levantamento utilizou-se dos seguintes termos e operadores booleanos: Taxonomia Corporativa; "Taxonomia corporativa"; Taxonomia AND corporativa; "Taxonomia AND corporativa"; Taxonomia OR corporativa; "Taxonomia OR corporativa"; Taxonomia facetada; "Taxonomia facetada"; Taxonomia AND facetada; "Taxonomia AND facetada"; Taxonomia OR facetada; "Taxonomia OR facetada"; Taxonomias corporativas; "Taxonomias Corporativas"; Taxonomias AND Corporativas; "Taxonomias AND Corporativas"; Taxonomias OR Corporativas; "Taxonomias OR Corporativas"; Taxonomias Facetadas; "Taxonomias Facetadas"; Taxonomias AND Facetadas; "Taxonomias AND Facetadas"; Taxonomias OR Facetadas; “Taxonomias OR Facetadas”. 


\section{Critérios de seleçáo}

Após o levantamento de dados com os termos mencionados foram obtidos 774 (setecentos e setenta e quatro) Teses e Dissertações, que levaram a uma análise minuciosa dos dados estabelecendo critérios para obter um resultado mais preciso. Em cada Tese e Dissertação foram analisados os seguintes critérios: Palavras-chave, título, resumo e objetivo. Essa análise proporcionou a seleção das Teses e Dissertações que se adequam a temática das Taxonomias Corporativas e Taxonomias Facetadas. Ao final foram totalizadas sete dissertações, não sendo recuperadas quaisquer documentos do tipo tese. Para a análise dos trabalhos recuperados foi utilizado o método da Análise de Conteúdo, a partir das seguintes categorias: objetivo, metodologia, produto final e trabalhos futuros.

\section{Apresentação e anÁlise dos Resultados}

Aqui estão apresentadas as análises realizadas em cada um dos documentos recuperados: as dissertações defendidas em programas de pós-graduação stricto sensu na Ciência da Informação. Ressalta-se que trata-se de parte dos resultados de uma pesquisa com escopo mais amplo, ainda em andamento.

\begin{tabular}{|l|l|}
\hline Dissertação 1 & Autor(a): AGANETTE, Elisângela Cristina \\
\hline $\begin{array}{l}\text { Título: Taxonomias corporativas: um estudo sobre definições e etapas de construção } \\
\text { fundamentado na literatura publicada }\end{array}$ \\
\hline Análise: Aganette (2010) possui como objetivo principal analisar a literatura publicada sobre definições \\
e métodos de taxonomias corporativas com a finalidade de definir este construto e conhecer os métodos \\
voltados à sua construção, para alcançar esse objetivo a autora define como metodologia a análise de \\
30 (trinta) definições encontradas na literatura e de 11 (onze) métodos para a construção de taxonomias \\
que foram analisados segundo as seguintes categorias: Tipo: origem; uso e elaboração; Essência: atri- \\
buto, classificação, estrutura e terminologia e Usos, Aplicações e Finalidades: potencialidades e gestão \\
da informação. Seu trabalho se caracteriza como exploratória e descritiva. Após realizar a análise das 30 \\
definições e dos 11 métodos, Aganette obteve como produto final duas propostas: uma de definição de \\
taxonomia e outra de 11 etapas de construção de taxonomias. Em sua seção de trabalhos futuros a autora \\
traz sugestões de pesquisas que ainda se fazem necessárias na área, como por exemplo: Verificar se as \\
práticas de elaboração de taxonomias levantadas na literatura dão condições para a construção de um \\
modelo de representação do conhecimento apto a ser aplicado na recuperação dos conteúdos informa- \\
cionais em ambientes corporativos; Propor a partir da verificação de aplicabilidade das etapas levantadas, \\
procedimentos específicos para cada etapa de construção da taxonomia, uma vez que se verifica ser essa \\
atividade inexistente nas práticas de construção atuais publicadas na literatura da área; Propor recomen- \\
dações para o processo de construção de taxonomias em ambientes corporativos.
\end{tabular}


TAXONOMIA CORPORATIVA E TAXONOMIA FACETADA: USOS E APLICAÇÕES...

\begin{tabular}{|l|l|}
\hline Dissertação 2 & $\begin{array}{l}\text { Autor(a): CAMARGo, Maria Fernanda Ma- } \\
\text { yer de }\end{array}$ \\
\hline Título: A construção de taxonomias para estruturação e recuperação de informações corporativas \\
\hline Análise: Camargo (2016) estabelece como objetivo principal a avaliação da a aplicação da Proposição \\
de Metodologia de Construção de Taxonomias Corporativas, elaborada do Aganette (2010) para a sua \\
validação em contexto organizacional. A autora caracteriza seu trabalho como qualitativa e participa- \\
tiva, para alcançar seu objetivo, Camargo estabelece como processo metodológico a análise do pro- \\
cesso de construção de uma Taxonomia Corporativa em uma empresa privada, a autora encaminhou \\
solicitações de análises das taxonomias às empresas e estabeleceu como critério: Maior quantidade \\
de termos no vocabulário controlado; Maior quantidade de categorias na estrutura taxonômica; Uso \\
constante da taxonomia pelos funcionários; Aperfeiçoamentos da estrutura taxonômica; Auditorias no \\
uso das taxonomias; Análise e validação da Proposição de Metodologia de Construção de Taxonomias \\
definida por Aganette (2010). Após a aplicação da Metodologia, o produto final de Camargo demons- \\
traram que as etapas baseadas em Aganette (2010) são factíveis de implementação e, que, ainda, \\
é o método mais extenso - são maiores as etapas do processo de implementação da construção de \\
taxonomias. Ao final, em seus trabalhos futuros a autora aborda sobre a necessidade da realização \\
de estudos que apontem os procedimentos de execução de cada uma das etapas propostas por Aga- \\
nette (2010), Camargo também sugere a elaboração de estudos de viabilidade para a construção de \\
ferramentas tecnológicas para a construção da estrutura taxonômica, em parceria com a Ciência da \\
Computação.
\end{tabular}

Quadro 2. Dissertação Camargo

\begin{tabular}{|l|l|}
\hline Dissertação 3 & $\begin{array}{l}\text { Autor(a): CAVALCANTE, Raphael da } \\
\text { Silva }\end{array}$ \\
\hline Título: Critérios para a avaliação de taxonomias navegacionais em sítios de comércio eletrônico \\
\hline Análise: 0 trabalho de Cavalcante (2012) possui como objetivo principal uma proposta de um con- \\
junto sistematizado de critérios para avaliação de taxonomias navegacionais, o autor caracteriza sua \\
dissertação como exploratória e quali-quantitativa. Para a metodologia o autor utilizou-se de amostras \\
das classes de produtos mais vendidos no varejo eletrônico brasileiro, realizou-se uma revisão biblio- \\
gráfica. Cavalcante utilizou-se dois critérios: Comunicabilidade e Organização. Para a execução da \\
análise considerou-se: (I) Análise dos níveis hierárquicos; (II) Utilização de facetas; e (III) Definição \\
de inconsistência. Para a avaliação das taxonomias seguiu-se a análise: (I) Descrição da categoria \\
analisada; (II) Descrição das inconsistências; e (III) Medidas ARC e AGT. Por fim o autor realizou uma \\
análise comparativa entre as lojas virtuais. Após seguir os critérios propostos na metodologia o autor \\
obteve como produto final a avaliação de que cada loja é representado por uma menção qualitativa. \\
Cavalcante diz que entende a construção de taxonomias como uma atividade subjetiva, mas defende \\
que recomendações mínimas devem ser consideradas, os critérios de avaliação foram validados, e \\
por fim o autor realiza 6 recomendações para implementação de ferramentas para a construção de \\
taxonomias navegacionais. Em suas propostas de trabalhos futuros o autor defende a exploração dos \\
diferentes tipos de taxonomia. No que se diz respeito às taxonomias navegacionais, sugere-se a con- \\
dução de trabalhos teóricos com vistas à consolidação do tema, bem como a análise de outros nichos \\
de aplicação, o que poderá resultar em novas recomendações para a construção das ferramentas e \\
em novos critérios de avaliação.
\end{tabular}

Quadro 3. Dissertação Cavalcante 


\section{Dissertação 4}

Autor(a):

MACULAN, Benildes Coura Moreira dos Santos

Título: Taxonomia Facetada Navegacional: construção a partir de uma matriz categorial para trabalhos acadêmicos

Análise: Maculan (2011) possui como objetivo criar uma taxonomia facetada navegacional a partir de uma matriz categorial, desenvolvida para trabalhos acadêmicos, visando a uma representação sistematizada e semântica do conteúdo de teses e dissertações capaz de facilitar a busca e a recuperação das informações. Para realizar sua pesquisa a autora estabelece como metodologia o uso da teoria da análise de domínio para a identificação do domínio, o uso do Modelo de Leitura Documentária, de Fujita e Rubi (2006), para o desenvolvimento da matriz categorial para trabalhos acadêmicos, e o método da análise de conteúdo, com a técnica da análise categorial temática, juntamente com os princípios da teoria da análise facetada de Ranganathan (1967). Maculan caracteriza seu trabalho como sendo de natureza empírica, exploratória, qualitativa, de pesquisa aplicada e bibliográfica. A utilização dessas teorias, modelos e métodos proporcionou como produto final o desenvolvimento da TAFNAVEGA e a validação do conjunto CAFTE. Os resultados demonstraram que o mecanismo TAFNAVEGA facilitou a tarefa de exploração, busca e recuperação de conteúdos dos documentos, dando acesso a dados tais como teorias, métodos e instrumentos de coleta de dados, possibilitando combinar essas diferentes informações. Em sua seção de trabalhos futuros, Maculan propõe a realização de cruzamento de dados entre metodologias e teorias utilizadas nas pesquisas, incluindo-se, também, as outras duas linhas de pesquisa da ECI-UFMG, enriquecendo a percepção sobre a natureza das práticas de pesquisa realizadas nessa Escola. A autora também aborda sobre a necessidade de um estudo do uso de um vocabulário controlado na indexação de documentos acadêmicos, a necessidade da criação de uma política de indexação que possa determinar o grau de exaustividade e especificidade para a indexação de documentos do tipo teses e dissertações e verificar a viabilidade da criação de um aplicativo tecnológico capaz de padronizar a entrada de dados (metadados) conforme a matriz categorial temática para trabalhos acadêmicos desenvolvida nesta pesquisa.

Quadro 4. Dissertação Maculan

\begin{tabular}{|l|l|}
\hline Dissertação 5 & Autor(a): MEDEIROS, José Wanderley \\
\hline Título: Taxonomia navegacional facetada: análise à luz dos princípios teóricos da classificação facetada \\
\hline $\begin{array}{l}\text { Análise: } 0 \text { objetivo principal de Medeiros (2013) é verificar se os princípios teóricos da classificação fa- } \\
\text { cetada encontram-se presentes nas taxonomias navegacionais facetadas de sítios de lojas virtuais e se } \\
\text { esses princípios podem contribuir para a elaboração dessas taxonomias. Para tal verificação, Medeiros } \\
\text { estabelece como metodologia a análise pelo modelo de Spiteri (1998), a análise de } 5 \text { lojas virtuais, atri- } \\
\text { buição de pontos nas taxonomias selecionadas e o total de pontos é levado em conta para a aferição do } \\
\text { índice de aderência. Em seu produto final o autor conseguiu identificar que a maioria dos princípios está } \\
\text { presente nas taxonomias analisadas, sobretudo aqueles relacionados à escolha e ordem das facetas e } \\
\text { apresentou-se um conjunto de recomendações para elaboração de taxonomias navegacionais faceta- } \\
\text { das com base nos princípios da classificação facetada. Medeiros propõe em seus trabalhos futuros a } \\
\text { elaboração de taxonomia navegacional facetada com aplicação das recomendações realizadas neste } \\
\text { trabalho e teste de navegação feita por usuários e a elaboração de material instrucional para elaboração } \\
\text { de taxonomias navegacionais facetadas, reunindo a teoria e a prática, expondo os princípios de maneira } \\
\text { mais clara e accessível para usuários leigos. }\end{array}$ \\
\hline
\end{tabular}




\begin{tabular}{|l|l|}
\hline Dissertação 6 & $\begin{array}{l}\text { Autor(a): MENEZES, Maria do Carmo } \\
\text { Vianna de }\end{array}$ \\
\hline $\begin{array}{l}\text { Título: Web semântica e o governo eletrônico brasileiro: revisão histórica e teórico-conceitual da } \\
\text { representação descritiva e estudo acerca da taxonomia e adoção de metadados na representação da } \\
\text { informação }\end{array}$ \\
\hline $\begin{array}{l}\text { Análise: Menezes (2010) propõe como objetivo principal analisar se a associação entre esse padrão } \\
\text { de metadado (e-PMG) à taxonomia para navegação (LAG/VcGE) permitirá a representação para a } \\
\text { recuperação da informação. Sua metodologia consiste em realizar uma entrevista com o coordenador } \\
\text { do segmento pesquisado e analisar a estrutura da taxonomia para navegação e as relações de simila- } \\
\text { ridade e complementaridade entre o e-PMG e o Dublin Core. Menezes caracteriza sua pesquisa como } \\
\text { sendo documental, pré-exploratória e exploratória, estudo de caso, descritiva, observação sistemática, } \\
\text { quali-quantitativa. Após a aplicação da metodologia a autora relatou em seu produto final que a maioria } \\
\text { dos princípios está presente nas taxonomias analisadas e os resultados obtidos indicaram que a as- } \\
\text { sociação entre o e-PMG e a LAG/VGGE trará benefícios significativos para a descrição e representação } \\
\text { da informação do portal do Governo eletrônico brasileiro foi apontado que a estrutura hierárquica e a } \\
\text { relação entre os termos da LAG/VCGE mantêm coerência com o referencial teórico adotado, no que se } \\
\text { refere ao dinamismo de atualização dos termos, ao controle do vocabulário e ao estabelecimento das } \\
\text { relações semântica. Para os trabalhos futuros a autora propõe um espaço para estudos correlatos, e } \\
\text { o prosseguimento desta pesquisa, sugerindo-se verificar se após a sua implementação, o padrão de } \\
\text { metadado, juntamente com a taxonomia adotada, descreve e representa a informação governamental } \\
\text { de forma adequada a possibilitar as solicitações de busca dos cidadãos. }\end{array}$ \\
\hline
\end{tabular}

Quadro 6. Dissertação Menezes

\begin{tabular}{|l|l|}
\hline Dissertação 7 & Autor(a): VITAL, Luciane Paula \\
\hline Título: Recomendações para construção de taxonomia em portais corporativos \\
\hline Análise: Vital (2007) propõe recomendações para o processo de construção de taxonomias em por- \\
tais corporativos como objetivo principal. A autora caracteriza sua pesquisa como qualitativa e explo- \\
ratória. A metodologia realizada pela autora consiste na identificação e seleção de práticas de ela- \\
boração ao desenvolvimento de taxonomias, identificação e seleção de funcionalidades discutidas na \\
literatura e relacionadas à representação e organização da informação em portais corporativos, análise \\
das etapas de desenvolvimento de taxonomias, e estabelecimento de princípios para a construção da \\
taxonomia em portais corporativos. O produto final obtido proporcionou apresentar proposições emba- \\
sadas nas áreas de Classificação, Indexação, Linguística e Documentação, cujas discussões teóricas \\
e metodológicas auxiliam o processo de construção de uma taxonomia, e o estabelecimento de orien- \\
tações para o processo de construção de taxonomias corporativas em 5 etapas. Vital sugere em seus \\
trabalhos futuros que novas pesquisas que busquem aprofundar outras etapas do desenvolvimento \\
de taxonomias não apresentadas neste trabalho, sugere também a aplicação das proposições em um \\
estudo de caso, buscando testá-las e aperfeiçoá-las, e pesquisas que extrapolam o ambiente cor- \\
porativo, direcionando a construção da taxonomia para outras áreas, como grupos de pesquisas em \\
universidades.
\end{tabular}

Quadro 7. Dissertação Vital

Ao analisar-se o objetivo principal das sete dissertações, percebeu-se que $57 \%$ das pesquisas estão relacionadas às taxonomias navegacionais facetadas. Percebe-se que os trabalhos são distintos em seus objetivos, porém, possuem o mesmo objeto de pesquisa, as dissertações de Aganette e Vital são as que possuem a mesma finalidade, que seria a de propor métodos ou 
recomendações para construção de taxonomias corporativas. As dissertações de Camargo e Cavalcante possuem objetivos que se aproximam, pois abordam sobre critérios de avaliação, enquanto Camargo avalia a taxonomia corporativa na prática, Cavalcante propõe critérios para a avaliação das taxonomias navegacionais. As demais dissertações (Maculan, Medeiros e Menezes) trabalham com comparações e verificações de princípios teóricos.

Durante a análise da metodologia realizou-se uma divisão dos métodos, uma em que fala-se a respeito de como o autor caracterizou o trabalho e a outra a respeito dos métodos aplicados. Em relação aos métodos, observou-se o constante uso de análises de modelos e teorias anteriores para estabelecimento de criação de taxonomias ou para uma nova proposta de critérios para construção de taxonomias. Isso se vê presente nas dissertações de Aganette, Maculan e Medeiros. Os trabalhos de Camargo, Menezes e Vital se assemelham pelo fato de ambos trabalharem com análise, avaliação e estrutura das etapas de desenvolvimento de taxonomias, ou de taxonomias já aplicadas. A dissertação de Cavalcante é a que mais se afasta das demais, o autor utiliza-se de amostras das classes de produtos mais vendidos no varejo eletrônico brasileiro juntamente com uma revisão bibliográfica.

Em relação à classificação da pesquisa, observou-se a ocorrência de cinco pesquisas definidas como exploratória, que representa mais de $71 \%$ das pesquisas. Dessas cinco, a de Menezes denominou-se como pré-exploratória e exploratória, mostrando, assim, a preocupação do pesquisador em obter mais informações sobre o objeto que está pesquisando. As pesquisas mostraram-se divididas em qualitativas e Quali-quantitativo, sendo que três trabalhos se qualificaram como qualitativa (Camargo, Maculan e Vital) e três como Quali-quantitativo (Cavalcante, Medeiros e Menezes). Um dos trabalhos (Aganette) não qualificou a sua pesquisa dessa maneira. As dissertações de Camargo e Menezes classificaram a pesquisa como estudo de caso, sendo que o trabalho de Camargo empregou o método da pesquisa participativa, enquanto o trabalho de Menezes realizou uma observação sistemática. Outros métodos utilizados foram a análise de conteúdo, pesquisa empírica, aplicada e bibliográfica.

A análise do produto final verificou os resultados obtidos em cada pesquisa. As dissertações de Aganette, Cavalcante, Medeiro e Vital resultaram em propostas ou recomendações de requisitos para a construção de taxonomias corporativas ou taxonomias navegacionais facetadas. O trabalho de $\mathrm{Ca}$ margo resultou na análise positiva da avaliação das etapas de construção de taxonomias corporativas proposta por Aganette (2010), uma vez que os requisitos são factíveis de implementação. A dissertação de Maculan resultou no desenvolvimento da taxonomia denominada TAFNAVEGA, que teve por 
base os princípios da Teoria da Classificação Facetada. O trabalho de Menezes chegou ao resultado de que esses mesmos princípios estão presentes nas taxonomias analisadas, verificando uma relação positiva entre os elementos do padrão de metadados e-PMG e a estrutura taxonômica da LAG/VCGE, associação que possibilitará a representação da informação do Governo eletrônico brasileiro.

Os trabalhos futuros propostos pelos autores mostraram-se similares, e percebe-se que muitos desses autores sugeriram trabalhos para que as novas pesquisas se aprofundem na exploração das taxonomias corporativas e facetadas dentro da área da CI, evidenciando a necessidade de trabalhos teóricos sobre o objeto de estudo e um maior aprofundamento nas pesquisas sobre as etapas de construção e desenvolvimento de taxonomias. O trabalho de Medeiros sugeriu a elaboração de um material institucional para elaboração de taxonomias navegacionais facetadas. A dissertação de Maculan se difere das outras, pois a autora traz propostas relacionadas a políticas de indexação e uso de vocabulário controlado.

\section{Considerações Finais}

Os dados apresentados neste artigo fazem parte de uma análise inicial dos dados coletados até o momento, que estão sendo filtrados e trabalhados com maior rigor, sobretudo no que se refere às análises dos trabalhos futuros que, acredita-se, trará um panorama das tendências e lacunas de pesquisa na temática trabalhada. Conforme foi apresentado, a produção dos pesquisadores da área mostra-se muito dispersa e limitado a poucos trabalhos, pois somente sete dissertações brasileiras sobre as taxonomias corporativas e taxonomias facetadas foram publicadas na área da Ciência da Informação nos últimos quinze anos. E isto, representa pouco para uma área que busca consolidar-se como campo de pesquisa de instrumentos de representação da informação e do conhecimento, considerado por estudiosos da área, como campo emergente e que vem traçando sua trajetória para o amadurecimento (Mueller e Santana, 2003). Considera-se que com mais trabalhos teóricos e práticos sobre taxonomias corporativas e facetadas, assim como com o aprofundamento de seu desenvolvimento, espera-se uma maior consolidação do tema na CI e em outras áreas do conhecimento. 
Agradecimento às agências de fomento Fundação de Amparo à Pesquisa em Minas Gerais (FAPEMIG), ao Conselho Nacional de Desenvolvimento Científico e Tecnológico (CNPq), à Coordenação de Aperfeiçoamento de Pessoal de Nível Superior (CAPES) e à Pró-Reitoria de Pós-Graduação (PRPG) da Universidade Federal de Minas Gerais (UFMG) pelo apoio financeiro.

\section{REFERÊNCIAS}

Aganette, Elisângela Cristina. 2010. “Taxonomias corporativas: um Estudo Sobre Definições e Etapas de Construção Fundamentado na Literatura Publicada”. Dissertação de mestrado, Universidade Federal de Minas Gerais, Escola de Ciência da Informação.

Alvarenga, Lídia. 2000. "Alguns enunciados sobre a comunicação e o uso de fontes de informação entre pesquisadores brasileiros da área da educação”, en Comunicação cientifica, organizado por Suzana Pinheiro Machado Mueller y Edilenice Jovelina Lima Passos, 123-138. Brasília, DF: Departamento de Ciência da Informação.

Camargo, Maria Fernanda Mayer de. 2016. "A construção de taxonomias para estruturação e recuperação de informações corporativas”. Dissertação de mestrado, Universidade Federal de Minas Gerais, Escola de Ciência da Informação.

Cavalcante, Raphael da Silva. 2012. "Critérios para a avaliação de taxonomias navegacionais em sítios de comércio eletrônico”. Dissertação de mestrado, Universidade de Brasília, Faculdade de Ciência da Informação.

Cendón, Beatriz Valadares, Jeannette Marguerite Kremer e Bernadete Santos Campello. 2000. "Teses e Dissertações”, en Fontes de informação para pesquisadores e profissionais, editado por Bernadete Santos Campello, 121-128. Belo Horizonte: UFMG.

Cunha, Murilo Bastos da, Cordélia Robalinho de Cavalcanti. 2008. Dicionário de biblioteconomia e arquivologia. Brasília: Briquet de Lemos.

Fujita, Mariângela Spotti Lopes e Milena Polsinelli Rubi. 2006. "Um modelo de leitura documentária para a indexação de artigos científicos: princípios de elaboração e uso para a formação de indexadores”. Data GramaZero 7 (jun.) (3): 1-19.

Maculan, Benildes Coura Moreira dos Santos. 2011. "Taxonomia facetada navegacional: construção a partir de uma matriz categorial para trabalhos acadêmicos". Dissertação de mestrado, Universidade Federal de Minas Gerais, Escola de Ciência da Informação.

Medeiros, José Wanderley. 2013. "Taxonomia navegacional facetada: análise à luz dos princípios teóricos da classificação facetada”. Dissertação de mestrado, Universidade Federal de Santa Catarina, Centro de Ciências da Educação.

Menezes, Maria do Carmo Vianna de. 2010. "Web semântica e o governo eletrônico brasileiro: revisão histórica e teórica conceitual da representação descritiva e estudo acerca da taxonomia e adoção de metadados na representação da informação”. Dissertação de mestrado, Universidade Federal da Bahia, Instituto de Ciência da Informação. 
Mueller, Suzana Pinheiro Machado e Edilenice Jovelina Lima Passos. 2000. "Alguns enunciados sobre a comunicação e o uso de fontes de informação entre pesquisadores brasileiros da área da educação", en Comunicação científica, editado por Lídia Alvarenga, 123-138. Brasília: Departamento de Ciência da Informação.

Mueller, Suzana Pinheiro Machado e Maria Gorette Santana. 2003. "A Ciência da Informação no CNPq - fomento à formação de recursos humanos e à pesquisa entre 1994-2002". DataGramaZero - Revista de Ciência da Informação (4): 1-19. http://repositorio.unb.br/handle/10482/963

Ranganathan, Shiyali Ramamrita. 1967. Prolegomena to Library Classification. Bombay: A. P. House, Ed.

Spiteri, Louise. 1998. "A Simplified model for facet analysis: Ranganathan 101". Canadian Journal of Information and Library Science 23 (1-2): 1-30.

Terra, José Cláudio C. 2005. Gestão do Conbecimento: o grande desafio empresarial. Rio de Janeiro: Campus.

Vital, Luciane Paula. 2007. "Recomendações para construção de taxonomia em portais corporativos”. Dissertação de mestrado, Universidade Federal de Santa Catarina, Centro de Ciências da Educação.

Para citar este texto:

Lopes, Pâmela Tamires Dias, Elisângela Cristina Agnanette y Benildes Coura M. S. Maculan. 2020. "Taxonomia corporativa e taxonomia facetada: usos e aplicações na ciência da informação no Brasil”. Investigación Bibliotecológica: archivonomía, bibliotecología e información 34 (82): 159-173.

http://dx.doi.org/10.22201/iibi.24488321xe.2020.82.58062 